\section{Exuberant tufted folliculitis}

Paulo Müller Ramos ${ }^{1}$

Helio Amante Miot ${ }^{1}$

DOI: http:/ /dx.doi.org/10.1590/abd1806-4841.20197952

Dear editor,

A 40-year-old African-American man reported a 5-year history of pustules and crusts on the scalp. Physical examination revealed extensive atrophic patches of cicatricial alopecia and polytrichia (Figure 1). Dermatoscopy of a single tuft showed more than 30 hairs emerging from one follicular orifice (Figure 2). Dermatoscopic imaging of a tuft removed via punch reveals the hairs retained within the involved follicular units (Figure 3). Histopathological examination showed polytrichia, peri- and intrafollicular infiltrate with neutrophils, lymphocytes, and histiocytes, which were most intense in the upper half of the dermis. Therefore, we reached the diagnosis of folliculitis decalvans.

There is a controversy concerning whether tufted folliculitis (TF) is a specific disease, a subset of folliculitis decalvans, or a common manifestation of different causes of alopecia. The term was first used by Smith in 1978 to describe a case of a patient with cicatricial alopecia who presented with hair tufts associated with intense inflammatory signs and follicular pustules. Culture from scalp biopsy revealed growth of S. aureus. ${ }^{1}$ In this first article, the author already pointed out many similarities between that case and the one described by Brocq in 1888 as folliculitis decalvans.

TF is caused by clustering of adjacent follicular units due to a fibrosing process that happens when the infundibular epithelia of damaged follicles heal leading to the formation of a common infundibulum. Telogen hairs can not detach and stay retained within the involved follicular units. ${ }^{2}$ Several diseases have been described as

Received 10 December 2017.

Accepted 12 April 2018.

* Work conducted at the Department of Dermatology, Faculdade de Medicina, Universidade Estadual Paulista, Botucatu (SP), Brazil.

Financial support: None.

Conflict of interest: None.

1 Department of Dermatology, Faculdade de Medicina, Universidade Estadual Paulista, Botucatu (SP), Brazil.

MAILING AdDREss:

Paulo Müller Ramos

E-mail: dermato.paulo@gmail.com

@2019 by Anais Brasileiros de Dermatologia

(cc) BY-NC

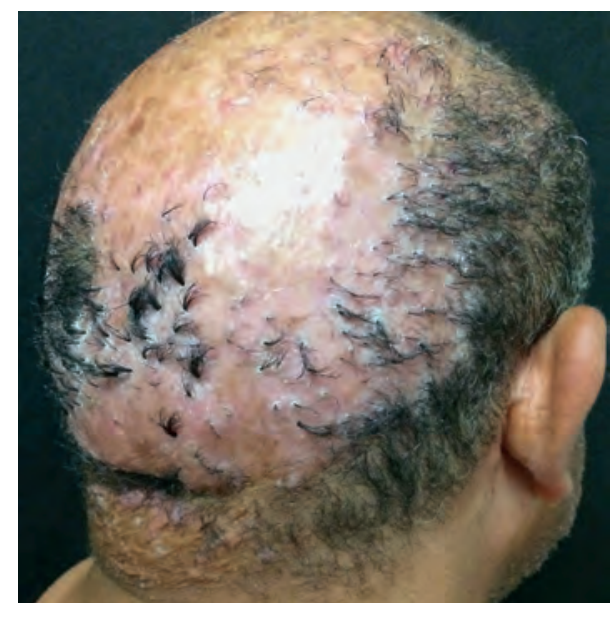

FIGURE 1:

Extensive areas

of cicatricial alopecia with polytrichia

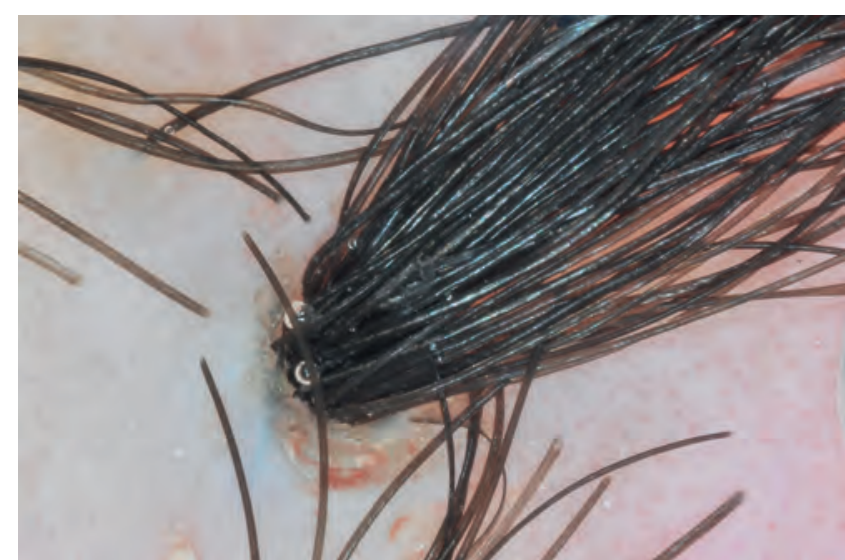

Figure 2: Dermoscopy shows multiple hairs emerging from the same follicular orifice

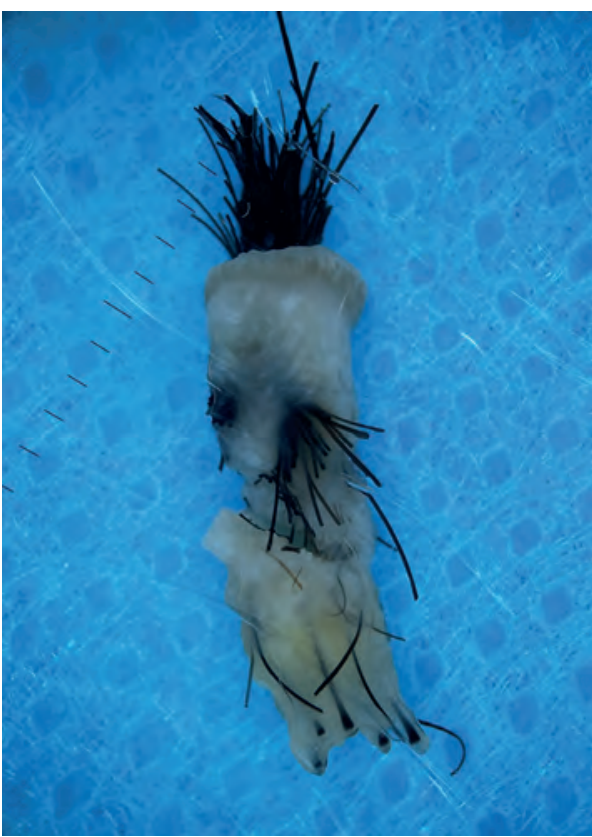

Figure 3: Dermatoscopic image of a tuft showing retained hairs within the involved follicular units 
cause of TF: folliculitis decalvans, central centrifugal cicatricial alopecia (CCCA), pemphigus vulgaris, folliculitis keloidalis, dissecting cellulitis, lichen planopilaris, discoid lupus, and tinea capitis. ${ }^{3}$ TF has already been described in a patient treated with cyclosporine and in two women having chemotherapy to treat breast cancer, one using lapatinib and the other, trastuzumab.

One cause of confusion is that most authors use the terms TF and polytrichia as synonyms. ${ }^{3}$ Polytrichia is defined as multiple ( 5 or more) hairs emerging from the same follicular opening. By defining TF only according to this pattern, it is easy to understand it just as a final stage of different diseases. Under this perspective, the use of the two terms for the same condition is a cause for confusion. The term TF could be abolished from the medical vocabulary in favor of polytrichia, which is semantically more appropriate.

However, the authors who see TF as a specific diagnosis understand that the term could not be used for all the patients with polytrichia, but should be reserved just for those cases of inflamed hair scalp with pustules that grow S. aureus (as in Smith's original description). As such, TF would be a subset of folliculitis decalvans. ${ }^{4}$

Despite this controversy, TF is frequently observed in patients with folliculitis decalvans. In a retrospective multicenter review study with 82 patients with folliculitis decalvans, $88 \%$ of them presented with TF. ${ }^{5}$ Huge tufts with more than 10 hairs are characteristic of this condition.

\section{REFERENCES}

Smith NP. Tufted folliculitis of the scalp. J R Soc Med. 1978;71:606-8.

2. Annessi $G$. Tufted folliculitis of the scalp: a distinctive clinicohistological variant of folliculitis decalvans. Br J Dermatol. 1998;138:799-805.

3. Sperling LC, Cowper S, Knopp EA. An Atlas of Hair Pathology with Clinical Correlations. 2nd ed. Boca Raton: CRC Press; 2012.

4. Powell J, Dawber RP. Folliculitis decalvans and tufted folliculitis are specific infective diseases that may lead to scarring, but are not a subset of centra centrifugal scarring alopecia. Arch Dermatol. 2001;137:373-4.

5. Vañó-Galván $S$, Molina-Ruiz AM, Fernández-Crehuet $P$, Rodrigues-Barata AR, Arias-Santiago S, Serrano-Falcón C, et al. Folliculitis decalvans: a multicentre review of 82 patients. J Eur Acad Dermatol Venereol 2015; 29:1750-7.

\section{AUTHORS'CONTRIBUTIONS}

Paulo Müller Ramos (iD) ORCID 0000-0002-1561-414X

Approval of the final version of the manuscript; Conception and planning of the study; Elaboration and writing of the manuscript; Obtaining, analyzing and interpreting the data; Intellectual participation in propaedeutic and/or therapeutic conduct of the cases studied; Critical review of the literature; Critical review of the manuscript Helio Amante Miot iD ORCID 0000-0002-2596-9294

Approval of the final version of the manuscript; Elaboration and writing of the manuscript; Critical review of the literature; Critical review of the manuscript

How to cite this article: Ramos PM, Miot HA. Exuberant tufted folliculitis. An Bras Dermatol. 2019;94(1):115-6.
Follicular psoriasis: an underdiagnosed entity?*

Bruno de Castro e Souza ${ }^{1}$

Luisa Groba Bandeira ${ }^{1}$

Thais do Amaral Carneiro Cunha ${ }^{1}$

Neusa Yuriko Sakai Valente ${ }^{1}$

DOI: http:/ / dx.doi.org/10.1590/abd1806-4841.20197987

Dear Editor,

Psoriasis is one of the most common inflammatory dermatoses and, in most patients, presents with erythematous scaling plaques on extensor areas. Classically, several clinical subtypes are described, such as plaque, inverted, guttate, palmoplantar, erythrodermic, and pustular. A minor variant discussed and reported is follicular psoriasis (FP), with only 25 reports in the international literature to date. Thus, the present article describes the first Brazilian case and revises concepts of this little known entity.

A 58-year-old female diabetic type 2 patient presented with erythematous, keratotic, exclusively follicular non-pruritic papules measuring $2-3 \mathrm{~mm}$ in diameter. The lesions were generalized, but with higher density in the gluteal and proximal regions of the thighs (Figures 1 and 2). Still, few isolated follicular pustules could be seen. Palms of the hands and soles of the feet were unchanged. The lesions appeared 4 years ago in the lower limbs with later gradual spread. Diagnostic hypotheses were pityriasis rubra pilaris (PRP) and keratosis pilaris with secondary infection. Previous histopathological examination of biopsy specimens revealed a diagnosis of follicular porokeratosis. Histopathological review showed parakeratosis and numerous intact and degenerate neutrophils within the lumens and in the epithelium of the follicular isthmus, where they formed a spongiform pustule. In addition, mild regular perifollicular acanthosis was also present (Figure 3). Grocott's staining

Received 16 December 2017.

Accepted 19 April 2018.

* Work conducted at Hospital do Servidor Público do Estado de São Paulo, São Paulo (SP), Brazil.

Financial support: None.

Conflict of interest: None.

1 Department of Dermatology, Hospital do Servidor Público do Estado de São Paulo, São Paulo (SP), Brazil.

MAILING AdDRESS:

Bruno de Castro e Souza

E-mail: brunocastro1990@hotmail.com

C2019 by Anais Brasileiros de Dermatologia 